\title{
Twiss Functions and Beam Sizes of the RHIC Insertion
}

\author{
S. Tepikian \\ April, 1994
}

The twiss functions and beam profiles of the RHIC insertion are presented. Figures 1-4 show the root of the beta functions in both the radial, $x$, and vertical, $y$, planes as well as the radial dispersion. Furthermore, a schematic of the beam line is shown scaled along the beam direction. The bars above the line are the focusing quadrupoles while those below the line denote defocusing, the larger boxes denote the dipoles and the smaller boxes represent the sextupoles. Additional information can be found in the subtitle. These four figures differ in the choice of $\beta^{*}=1,2,6$ and $10 \mathrm{~m}$ respectively, which is the beta function at the crossing point given at $S=0$.

Figure 5 is an expanded view of the injection region with $\beta^{*}=10 \mathrm{~m}$, the insertion optics for injection.

Figures 6 and 7 show the twiss function and beam, size through the triplets and crossing dipoles. The insertion optics is tuned to $\beta^{*}=1 \mathrm{~m}$ which will be the case for storage mode. Similarly, Figures 8 and 9 are for $\beta^{*}=10 \mathrm{~m}$. The beam size is calculated from:

$$
\sigma_{x, y}=\sqrt{\frac{\varepsilon_{N} \beta_{x, y}}{6 \pi(\beta \gamma)}}
$$




\section{RHIC Insertion Functions}

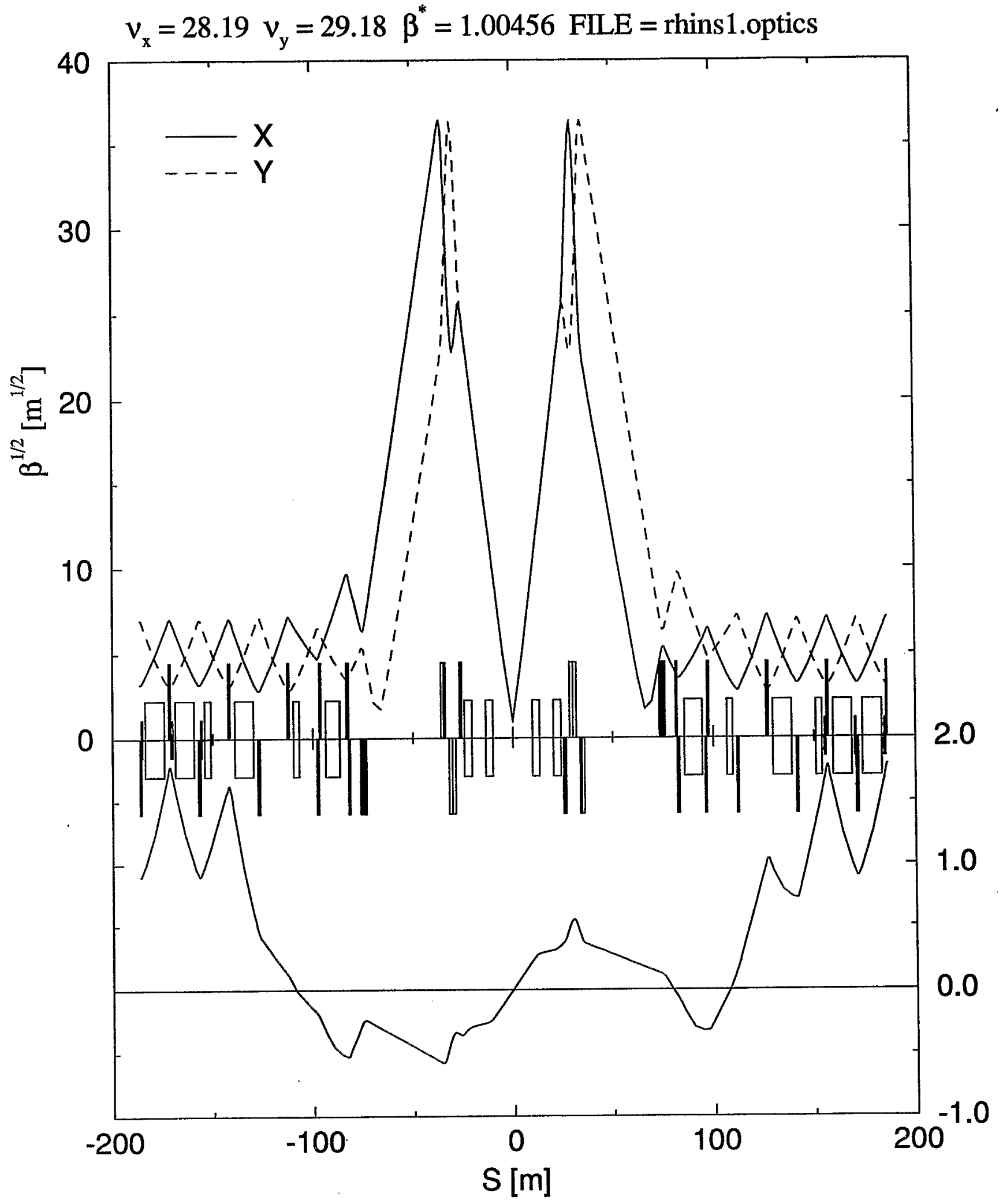

Figure I

Time: Thu Apr 21 11:23:55 1994 Last file modily time: Thu Apr 21 11:18:24 1994 
RHIC Insertion Functions

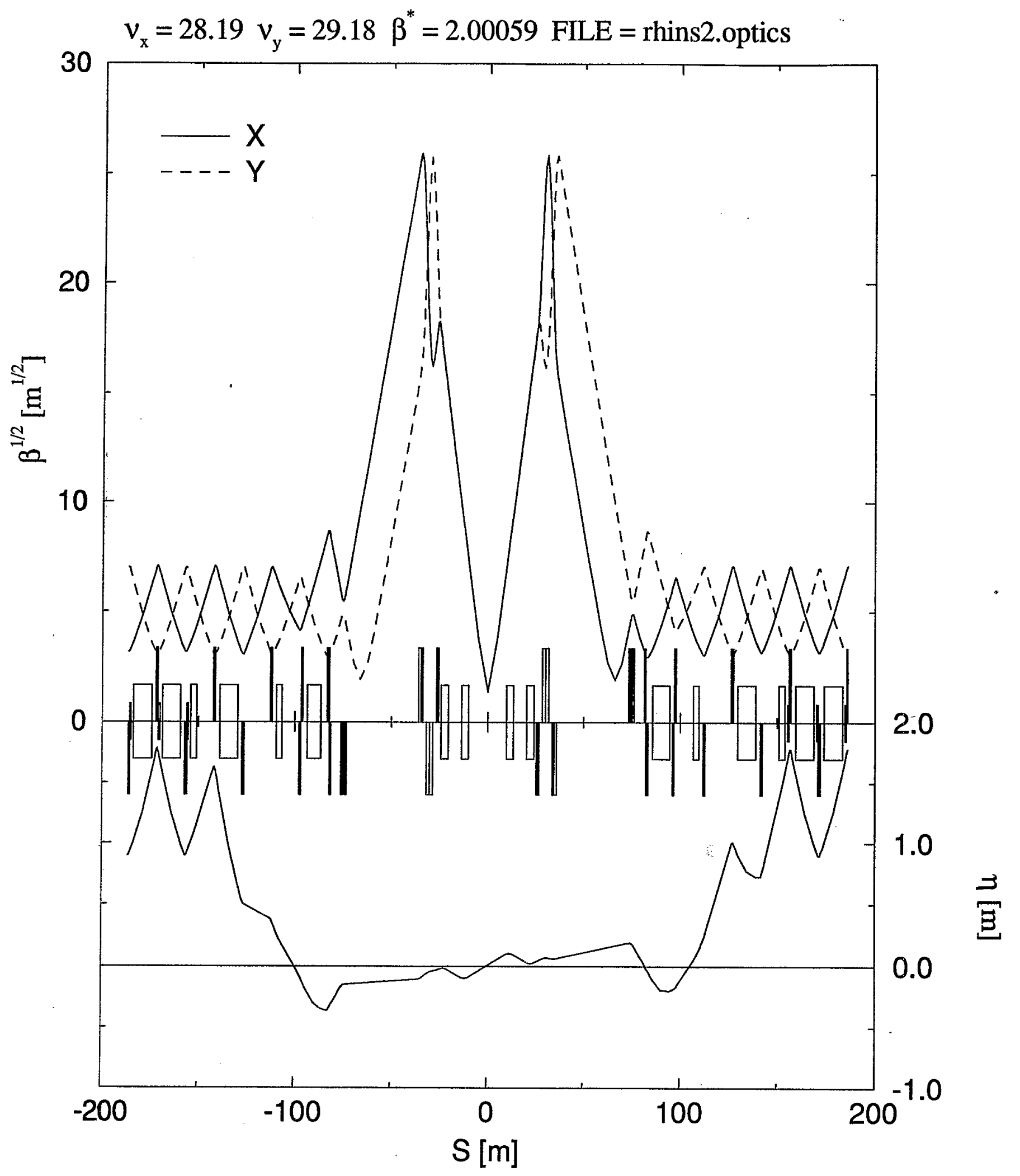

Figure 2

Time: Tru Apr 21 11:24:17 1994 Last file modify time: Thu Apr 21 11:18:54 1994 
RHIC Insertion Functions

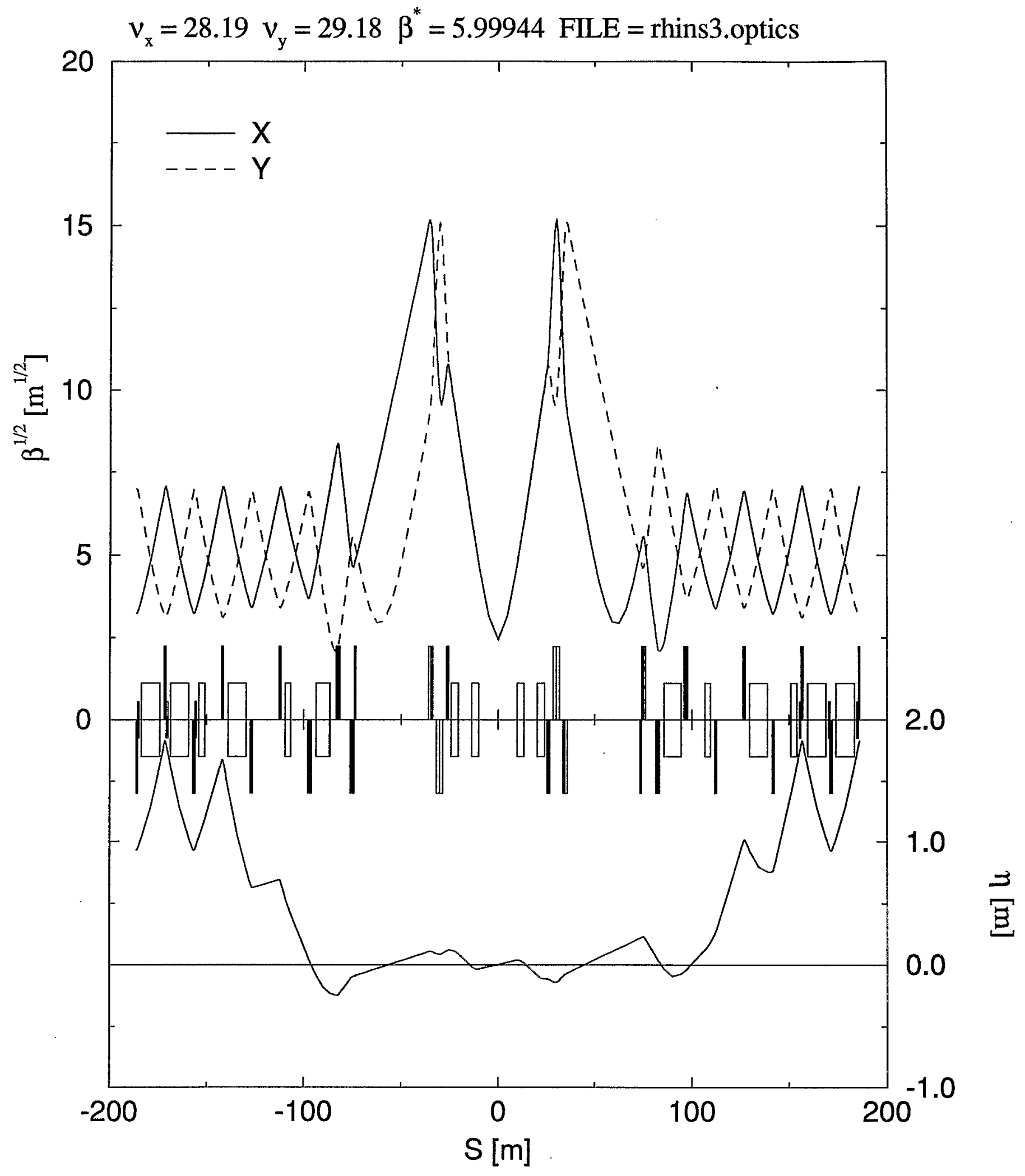

Figure 3 


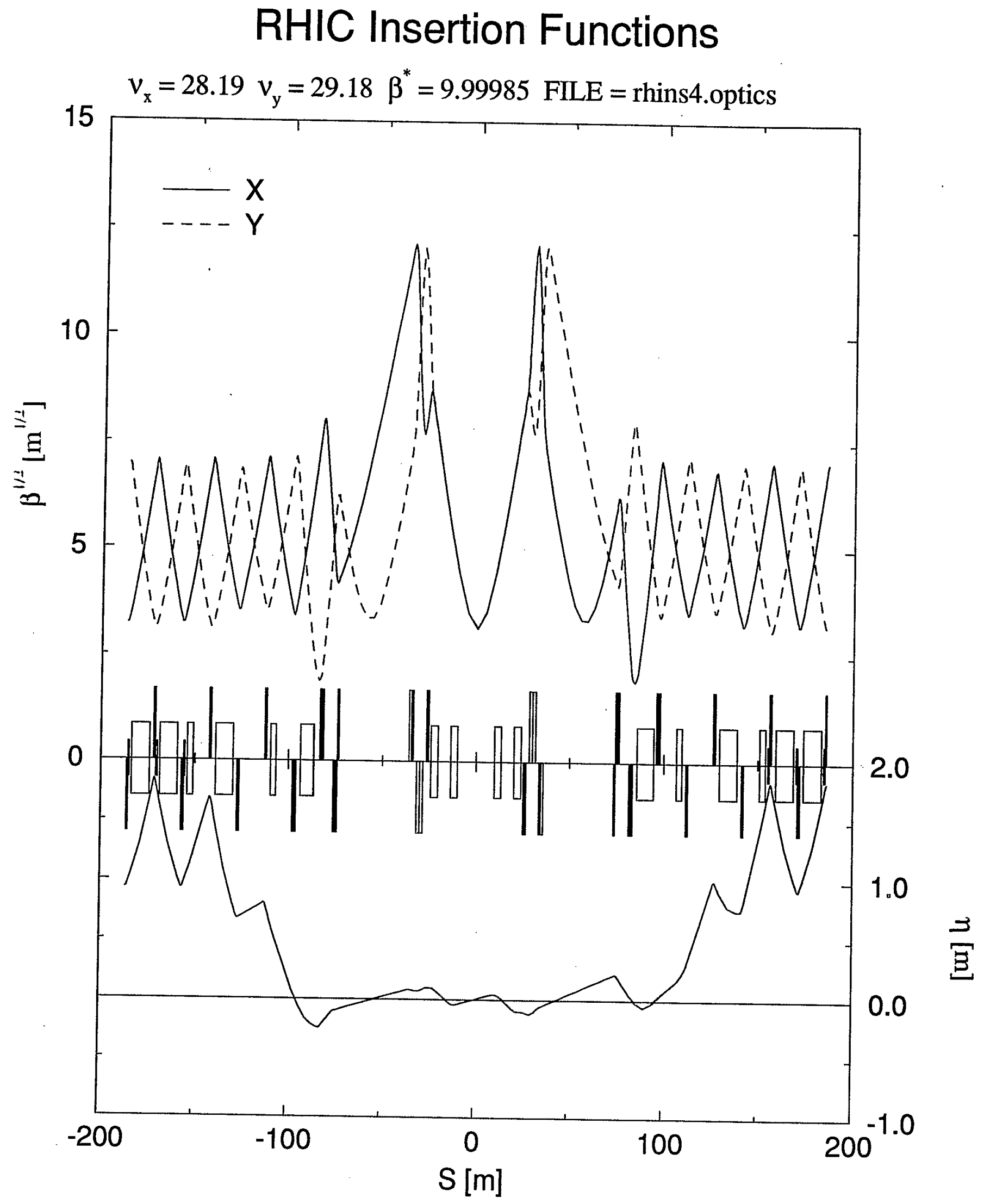

Figure 4 


\section{RHIC Insertion Functions}

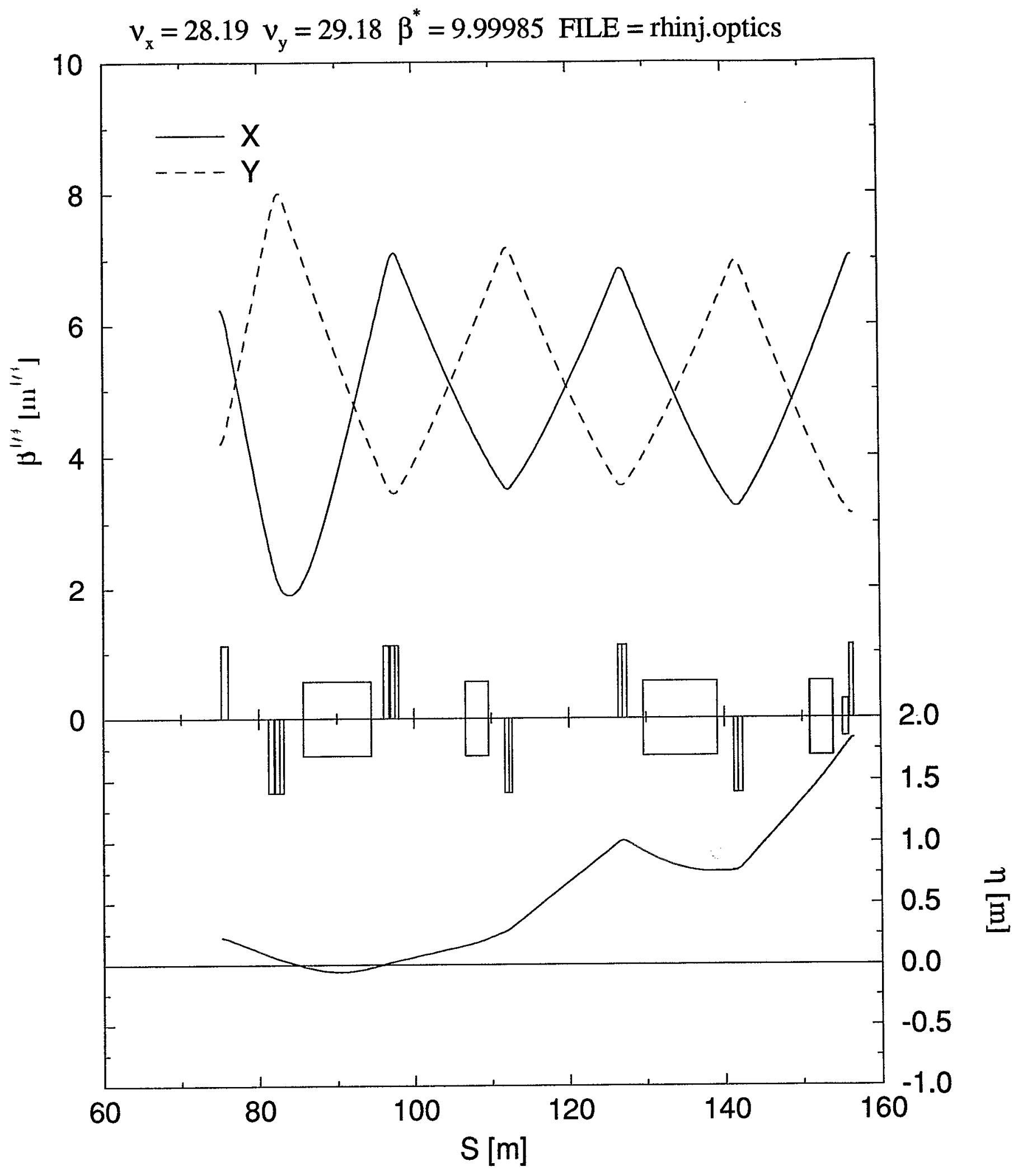

$F_{\text {Igna }} 5$ 


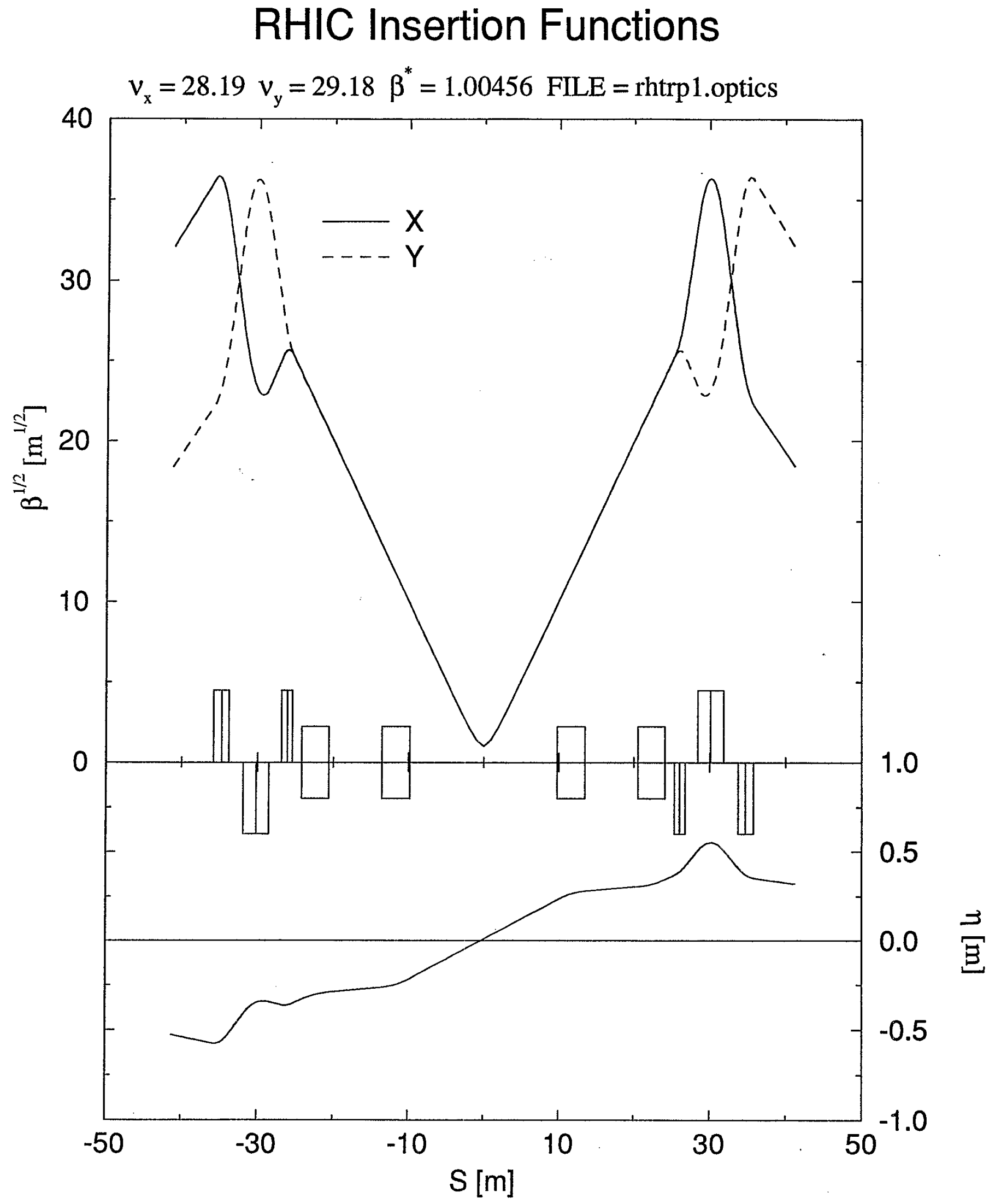

Figure 6 


\section{RHIC Beam Profiles}
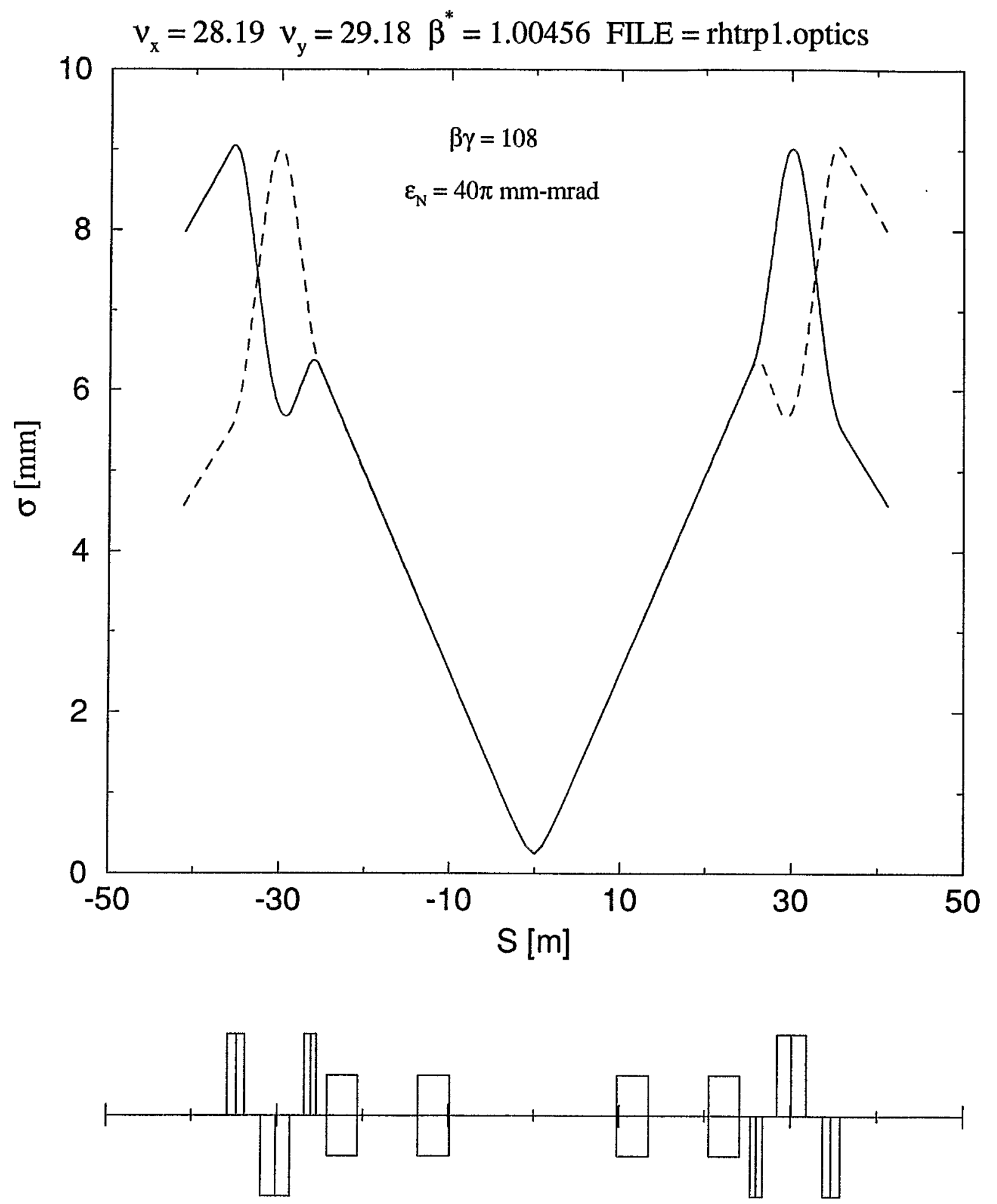

Figure 7 


\section{RHIC Insertion Functions}

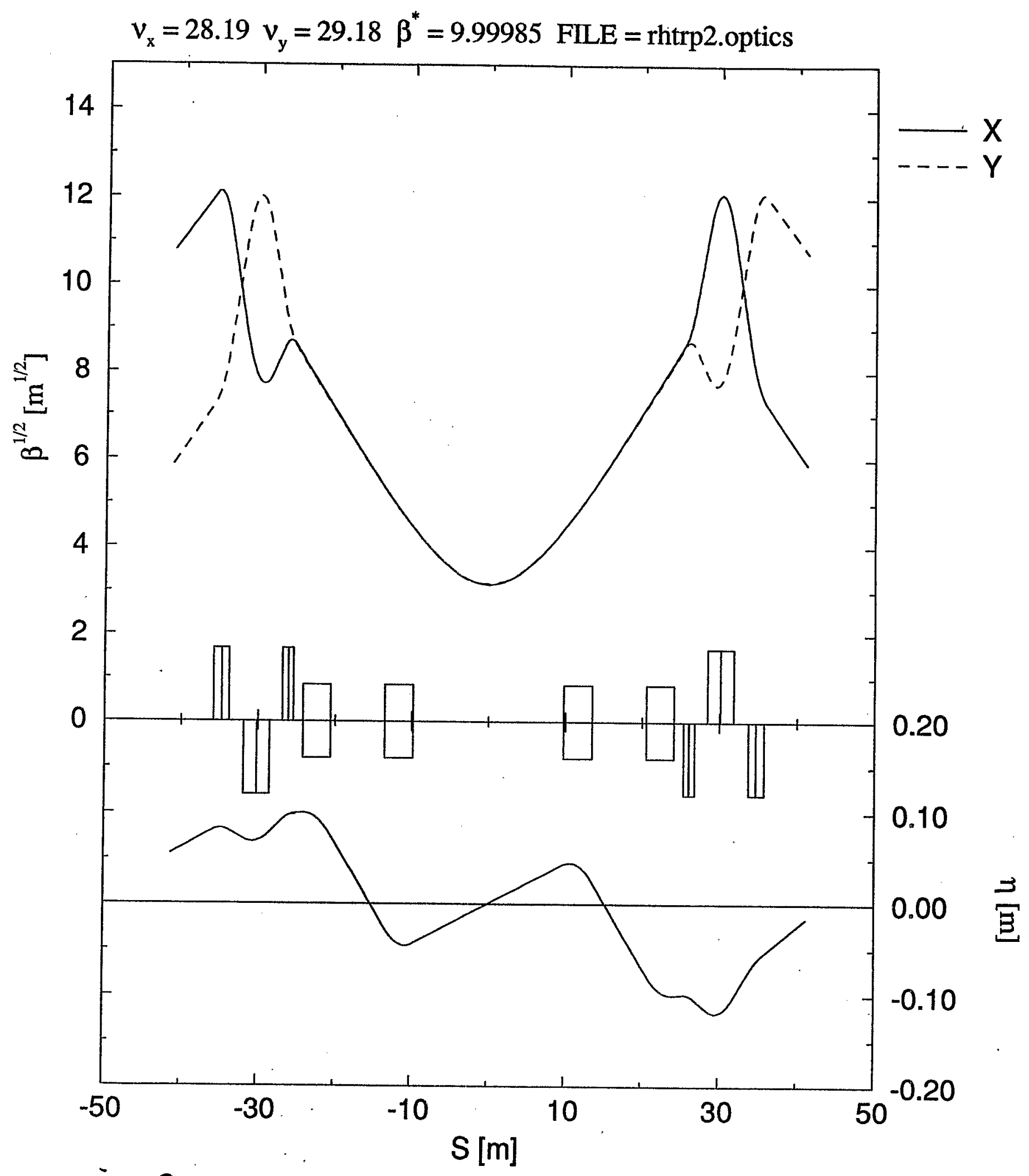

Figure 8 


\section{RHIC Beam Profiles}

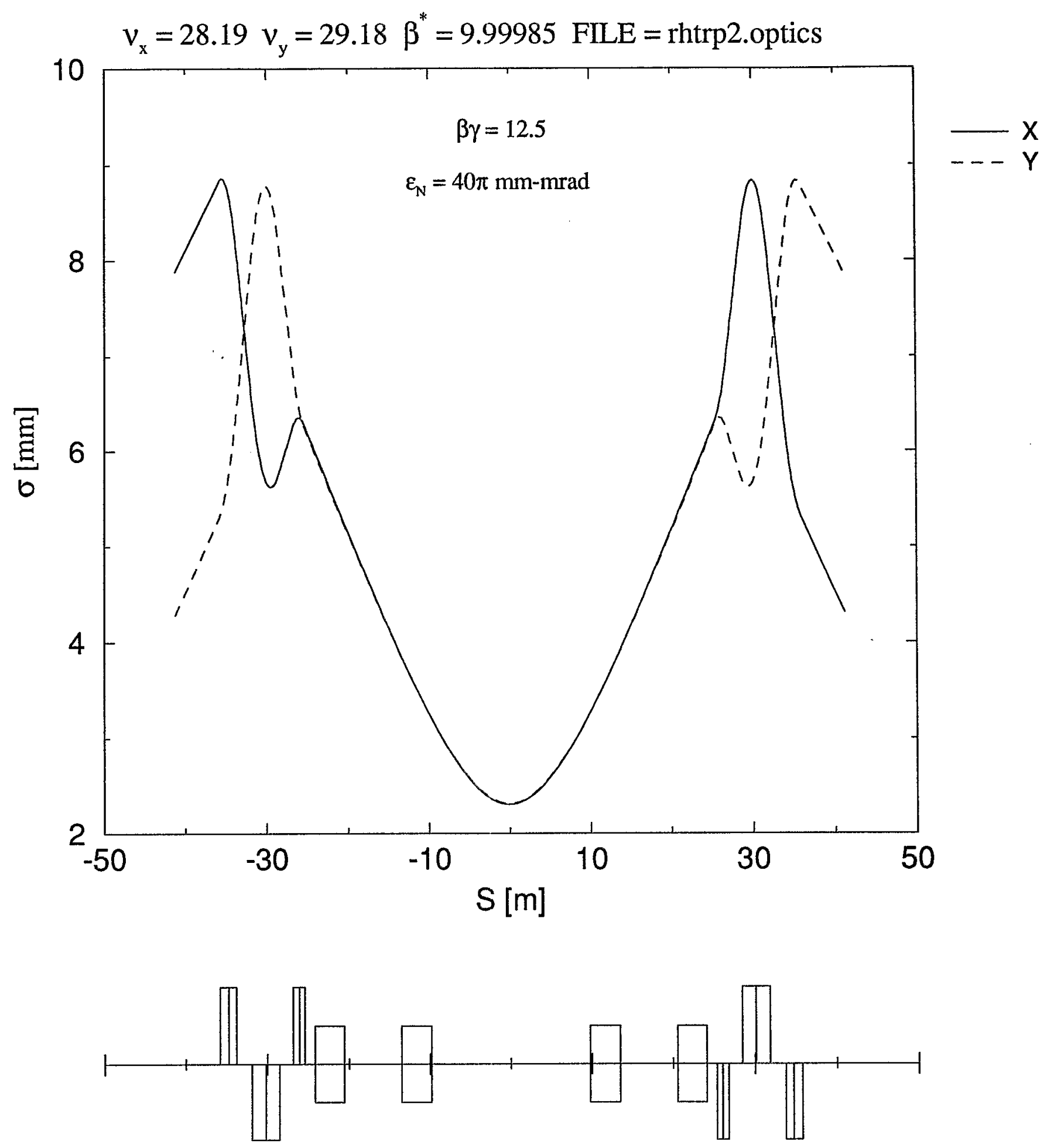

Figure 9

Time: Fri Apr 22 14:07:28 1994 Last file modify time: Fri Apr 22 11:13:04 1994 